\title{
List of Reviewers and Editors
}

Reviewers ensure the rigorous standards of the scientific process by taking part in the peer-review system, uphold the integrity of the journal by identifying invalid research and helping to maintain the quality of the journal.

We express our deep gratitude to them for their painstaking and sincere effort.

\begin{tabular}{|l|l|}
\hline Name & Discipline \\
\hline Professor M Jalal Uddin & Community Medicine \\
\hline Professor Sirajun Noor Rosy & Obstetrics \& Gynaecology \\
\hline Professor Nahid Sultana & Microbiology \\
\hline Dr. A K M Zafarullah & Pediatrics \\
\hline Dr. Razia Sultana & Pathology \\
\hline Dr. Jahangir Alam Chowdhury & Physical Medicine \\
\hline Dr. Md. Gias Uddin & Psychiatry \\
\hline Dr. Asma Mostafa & Anatomy \\
\hline Dr. Farah Naz Mabud & Obstetrics \& Gynaecology \\
\hline Dr. Monira Khatun & Physiology \\
\hline Dr. Rajat Sankar Roy Biswas & Medicine \\
\hline $\begin{array}{l}\text { Dr. Chinmoy Baidya } \\
\text { Dr. Mohammad Abu Bakar }\end{array}$ & Community Medicine \\
\hline
\end{tabular}

[This list is not according to seniority] 